\title{
Analisando a participação do Brasil e dos demais países da América Latina nos encontros do IETF
}

\author{
Juliao Braga ${ }^{1}$, Patricia Takako Endo ${ }^{2}$, Marcelo Santos ${ }^{3}$, Jéferson Campos Nobre ${ }^{4}$ \\ Leylane Graziele Ferreira da Silva ${ }^{2}$, Gleyson Rhuan \\ Nascimento Campos ${ }^{2}$, Nizam Omar ${ }^{5}$ \\ ${ }^{1}$ VRS at University of Saskatchewan \\ PPGEEC - UPM \\ São Paulo, SP \\ ${ }^{2}$ Universidade de Pernambuco \\ Caruaru - PE - Brasil \\ ${ }^{3}$ Instituto Federal do Sertão Pernambucano \\ Salgueiro, PE - Brasil \\ ${ }^{4}$ Universidade do Vale do Rio dos Sinos \\ São Leopoldo, RS - Brasil \\ ${ }^{5}$ Universidade Presbiteriana Mackenzie \\ São Paulo, SP - Brasil \\ juliao@braga.net.br, patricia.endodupe.br \\ marcelo.santosdifsertao-pe.edu.br, jcnobredunisinos.br \\ leylanegraziele@gmail.com, gleysonrhuan@gmail.com, nizam.omar@mackenzie.br
}

\begin{abstract}
This paper discusses the participation of Brazilians in IETF meetings comparing with other countries in Latin America and the Caribbean. In addition, it describes the initiatives to incentivize participation in the development of documents and the movements that provide resources to finance such participations. It describes the Brazilian initiatives that stimulate participation and recommends others that once carried out in Brazil and other countries in Latin America and the Caribbean will increase the presence of more volunteers.
\end{abstract}

Resumo. Este artigo aborda a participação de brasileiros nos encontros do IETF comparando com outros países da América Latina e Caribe. Adicionalmente ele descreve as iniciativas de participação no desenvolvimento de documentos e os movimentos que disponibilizam recursos para financiar tais participações. Descreve as iniciativas brasileiras que estimulam a participação e recomenda outras que levadas a cabo no Brasil e em outros países da América Latina e Caribe aumentarão a presença de mais voluntários.

\section{Introdução}

O Internet Engineering Task Force (IETF) é uma comunidade internacional composta por milhares de pessoas que trabalham, voluntariamente, no processo de padronização dos protocolos da Internet. A forma como o IETF funciona, criando padrões abertos através 
do consenso, são fundamentais para que a Internet funcione de forma estável e eficiente. Assim, através da colaboração de diversos membros organizados em grupos de trabalhos (WG - working groups) temos, por exemplo, a padronização de protocolos como o TCP, IPv4 e IPv6 que são fundamentais para o funcionamento de Internet. Nesse contexto é importante a participação de membros brasileiros e da América Latina objetivando a troca de conhecimento entre países emergentes, além de participação na construção de uma rede cada vez melhor.

Este artigo tem como principal objetivo, analisar, sob a ótica da participação nas reuniões do IETF, a efetividade das ações realizadas nos países da América Latina e Caribe na direção de identificar o que estimula a participação nestes encontros e também delinear novas formas de fomentar a participação de novos membros. Para tanto, foram analisados dados relacionados à inscrição nos encontros do IETF deste a segundo encontro de 2008 (IETF 72) até o último de 2016 (IETF 97). Os resultados mostram que dentro da América Latina, o Brasil tem se destacado pelo crescente número de participações nas reuniões presenciais da IETF, sendo o país com maior número de participantes.

O restante deste artigo está organizado da seguinte forma: na seção 2 é apresentada uma descrição geral da ISOC, IETF, e outras organizações relacionadas ao tema do trabalho. Na seção 3 estão descritos como e de onde os dados aqui utilizados foram obtidos e onde estão disponíveis. Na seção 4 tem-se uma descrição sobre a área de atuação do RIR que cuida dos recursos e facilidades da Internet na América Latia e Caribe. Na seção 6 serão tratados os resultados em forma de gráficos e tabelas. Na seção 7 estarão as considerações finais que concluem o trabalho adicionando, também, sugestões e recomendações para uma participação mais efetiva tanto no IETF como no Internet Research Task Force (IRTF).

\section{O Ecossistema da Infraestrutura da Internet}

O ecossistema da Internet é formado por um conjunto de stakeholders que buscam continuamente contribuir com melhorias para o seu funcionamento. Nesse cenário, o modelo de funcionamento da Internet evoluiu de uma pequena rede de pesquisa do governo americano, a ARPANET, para uma rede com bilhões de usuários sendo o seu funcionamento indispensável para diversos setores da economia de vários países.

Para organizar esse ecossistema complexo foram criadas diversas entidades com responsabilidades bem definidas para que haja então uma coordenação das atividades necessárias para o bom funcionamento da rede. Nesse contexto, podemos destacar algumas entidades que possuem papel fundamental no ecossistema da Internet: Internet Society (ISOC), Internet Engineering Task Force (IETF), Internet Corporation for Assigned Names and Numbers (ICANN), Internet Assigned Numbers Authority (IANA), Sistemas Autônomos (ASs) e um conjunto de Registros Regionais da Internet (RIRs). A Figura 1 exibe o relacionamento dessas entidades.

Endereços IPs (Internet Protocol) precisam ser gerenciados e atribuídos cuidadosamente para o bom funcionamento e estabilidade da Internet. O IANA é responsável por gerenciar sistemas de nomes de domínios (DNS) raízes, coordenar o número de sistema autônomos (ASs), delegar recursos para os RIRs, além de manter os nomes e números de protocolos desenvolvidos pela comunidade do IETF. Podemos ver o IANA como um conjunto de atividades relacionadas a nomeação e endereçamento na Internet, onde a 


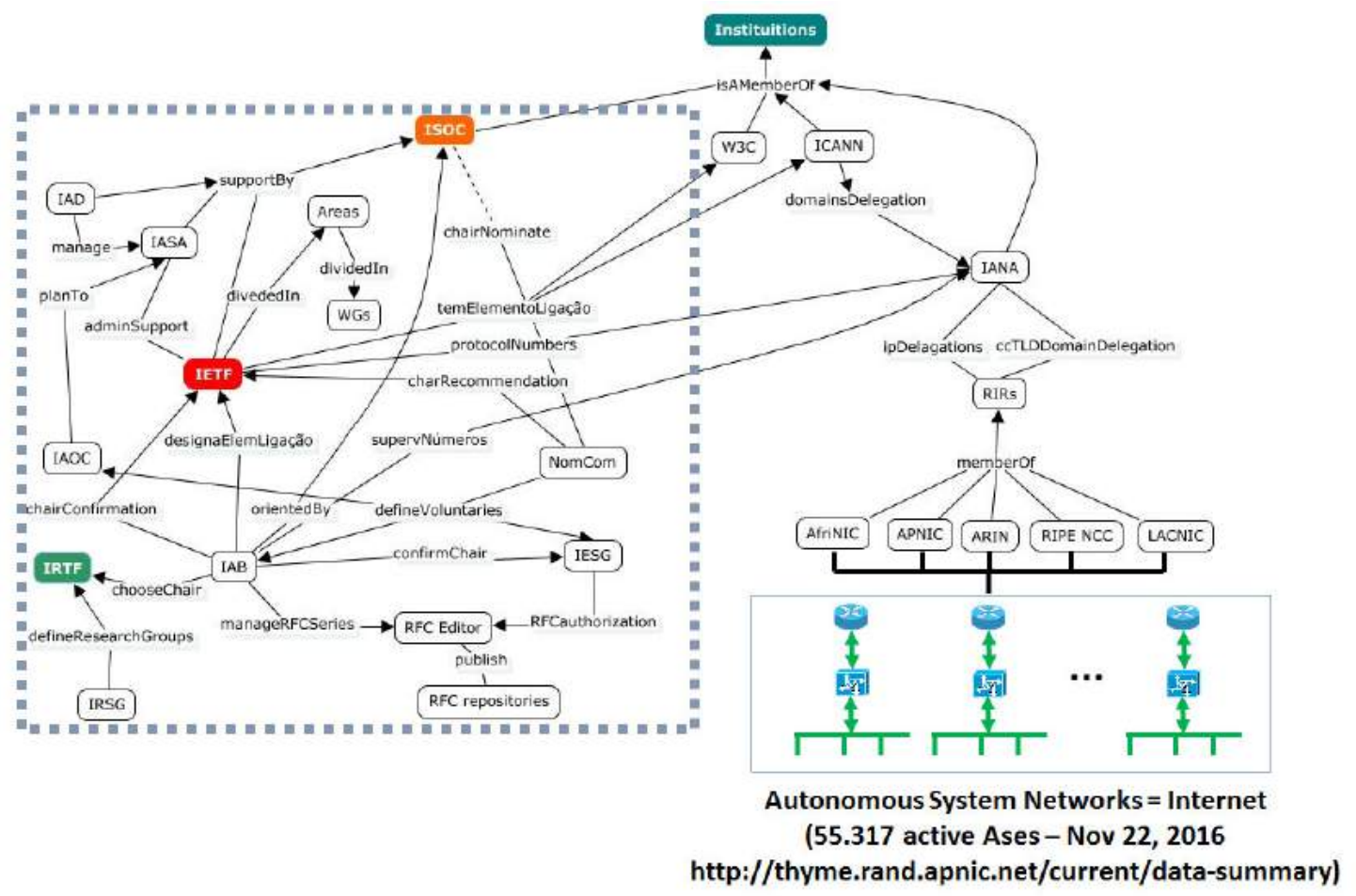

Figura 1. Mapa Conceitual do Ecosistema da Infraestrutura da Internet. Adaptado de [Braga and Omar 2014]

administração dessas tarefas e sua execução são realizadas pelo ICANN.

Numa escala regional, temos cinco RIRs: African Network Information Center (AFRINIC) ${ }^{1}$, Asia Pacific Network Information Centre (APNIC) ${ }^{2}$, American Registry for Internet Numbers (ARIN) ${ }^{3}$, Latin American and Caribbean Internet Addresses Registry (LACNIC) ${ }^{4}$ e o Réseaux IP Européens Network Coordination Centre (RIPE NCC) ${ }^{5}$. O papel básico de cada RIR é administrar e supervisionar números de espaço de endereço IP dentro de uma determinada região. Cada RIR tem sua própria política, mas devem obedecer políticas globais definidas pelo IANA.

Nesse cenário, entidades padronizadoras como o IETF/IRTF, World Wide Web Consortium (W3C), International Telecommunications Union (ITU-T) e Institute of Electrical and Electronics Engineers (IEEE) têm o importante papel de discutir e definir a criação de diversos protocolos que ajudam a Internet a funcionar. Simonelis [Simonelis 2005] descreve em maiores detalhes o relacionamento dessas entidades.

\subsection{Internet Society (ISOC)}

A ISOC foi uma entidade sem fins lucrativos que surgiu em 1993 com a ideia de mover toda discussão não técnica para fora do IETF [Huizer 1996]. Em resumo, o seu objetivo

\footnotetext{
${ }^{1}$ https://www.afrinic.net/

${ }^{2}$ https://www.apnic.net/

${ }^{3}$ https://www.arin.net/

${ }^{4}$ http://www.lacnic.net/en/web/lacnic/inicio

${ }^{5}$ https://www.ripe.net/
} 
é promover o desenvolvimento de padrões na Internet, bem como promover iniciativas educacionais e políticas públicas ligadas à Internet ${ }^{6}$.

Como o IETF foi criado em 1986, quando a ISOC surgiu, os seus membros eram pessoas fortemente ligadas ao IETF com o papel de gerenciar diversas ações para melhoria da Internet. Após o surgimento da ISOC, o IETF deixou de ser financiado pelo governo americano e passou a ser uma entidade sem fins lucrativos. Dessa forma, com a desvinculação do governo americano e sob gerenciamento da ISOC, o IETF popularizouse mundialmente sendo referência na definição e discussões de padrões para a Internet. A Figura 2 mostra resumidamente essa evolução histórica.

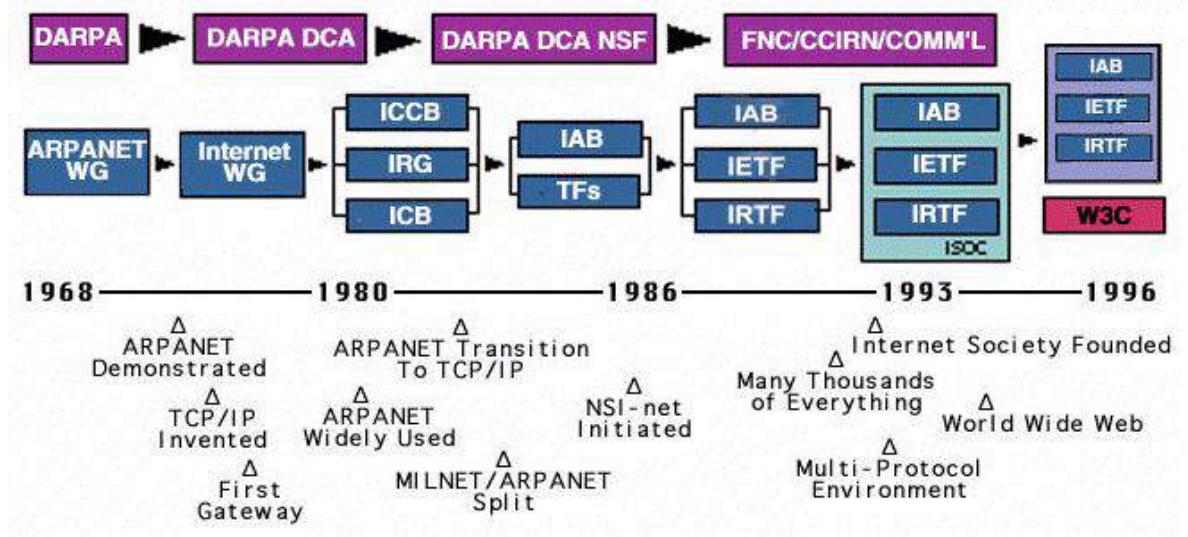

Figura 2. Linha do tempo e os principais movimentos que definiram o futuro da Internet. Fonte: [Leiner et al. 2009]

Segundo o próprio site da ISOC Brasil ${ }^{7}$, pode-se destacar as seguintes linhas de atuação da ISOC:

- Facilitar o desenvolvimento aberto de padrões, protocolos, gestão e infra-estrutura técnica da Internet;

- Suportar/apoiar a educação nos países em desenvolvimento especialmente e onde existir demanda;

- Promover desenvolvimento profissional e construir comunidades para ampliar a participação e a liderança em áreas importantes para o desenvolvimento da Internet;

- Prover informação confiável sobre a Internet;

- Prever fóruns de discussão em assuntos que afetam a evolução, desenvolvimento e os usos técnicos, comercial, empresarial etc da Internet;

- Fomentar a cooperação internacional e o intercâmbio entre comunidades e culturas, promovendo a autodeterminação;

- Centralizar/facilitar os esforços de cooperação em prol da Internet como uma ferramenta benéfica de todos;

- Prover gestão e coordenação para iniciativas estratégicas e esforços de disseminação humanitária, educacional da sociedade, dentre outros contextos;

\footnotetext{
${ }^{6} \mathrm{https}: / /$ www.isoc.org.br/post/escritorio-brasileiro/7

${ }^{7}$ https://www.isoc.org.br/post/escritorio-brasileiro/7
} 


\subsection{IETF e IRTF}

O IETF é uma grande comunidade internacional formada por voluntários interessados no desenvolvimento da Internet. Em resumo, a missão da IETF é tornar o funcionamento da Internet melhor [Alvestrand 2004] através do estabelecimento de padrões abertos por meio do consenso da comunidade que a compõe.

Atualmente, existem sete grandes áreas ${ }^{8}$ distintas (ex: segurança e roteamento) dentro do IETF onde são discutidos padrões que norteiam o funcionamento da Internet. Dentro de cada área existem grupos de trabalhos (Working Groups - WGs) que discutem e definem padrões para problemas específicos. As discussões ocorrem através de listas de e-mails e três encontros presenciais anuais. As reuniões presenciais tem como um de seus objetivos consolidar o que vem sendo discutido pelo voluntariado, nas listas de discussões por e-mail.

Dentro de cada WG há primeiro a criação de um proposta de padronização denominada I-D (Internet Draft) com um prazo de expiração para que ocorram as discussões para decidir se tal I-D irá virar realmente um padrão. Caso um I-D torne-se um padrão através do consenso da comunidade, então ele torna-se uma RFC (Request for Comments). Dessa forma, o processo de criação de uma RFC [Bradner 1996] é precedido por um Internet Draft (I-D) discutido amplamente dentro da comunidade da IETF. Alguns pontos devem ser levados em consideração nesta etapa :

- Priorização de implementação e código;

- Clareza, objetividade e facilidade de entendimento do documento;

- Maturidade técnica

- Atualidade do tema discutido.

A RFC 1543 ([Postel 1993]) define as regras e formatação para criação de uma RFC. Como exemplo, todo o texto deve ser escrito por meio de caracteres ASCII e cada página deve ser limitada a 58 linhas. É importante ressaltar que mesmo depois de definidas, RFCs sofrem constantes alterações com o passar do tempo, caso a comunidade ache conveniente atualizá-las.

De forma paralela ao IETF, existe o Internet Research Task Force (IRTF). O IRTF promove a discussão de pesquisas a longo prazo através de grupos de pesquisa (Research Groups), que funcionam de forma semelhante ao grupos de trabalho da IETF. A RFC 2014 [Weinrib and Postel 1996] define as diretrizes dos grupos de pesquisa do IRTF.

Podemos destacar alguns pontos que norteiam como o IETF e IRTF funcionam:

- Os padrões desenvolvidos não são impostos por empresas ou por questões políticas. Os padrões oriundos do IETF/IRTF são padrões utilizados voluntariamente.

- Cada área de trabalho possui duas pessoas responsáveis pela sua coordenação, aprovação e criação de WGs e BOFs (Birds of a Feather).

- O desenvolvimento de padrões ocorrem dentro de WGs por iniciativa dos participantes.

- Os I-D viram RFCs através do consenso entre a maioria dos participantes de um WG.

\footnotetext{
${ }^{8}$ https://www.ietf.org/iesg/area.html
} 
- A língua oficial é o inglês.

- Tanto os I-D quanto as RFCs devem ser escritas no formato ASCII.

- Todo documento produzido é público e pode ser desenvolvido colaborativamente por qualquer pessoa.

- Existem várias categorias de RFCs e nem toda RFC é necessariamente um padrão.

- Dentro do IETF os argumentos técnicos, código rodando e meritocracia são os pilares para seu funcionamento.

Adicionalmente, o TAO da IETF [Hoffman and Harris 2006] fornece uma visão de como o ecossistema da Internet funciona juntamente com o documento criado pela ISOC ${ }^{9}$.

\section{Metodologia}

Como dito anteriormente, o IETF realiza três encontros presenciais anuais em diferentes países. Desde o segundo encontro de 2008, o IETF vem disponibilizando na web a relação dos inscritos em seus encontros. Um programa em PHP captura estas informações e agrupa em um banco de dados todas as informações disponíveis nas URLs https://www.ietf.org/registration/ietf $\underline{x} /$ attendance.py, onde $72 \leq \underline{\mathbf{x}} \geq$ encontro atual. Um outro programa exibe o conteúdo desta base ${ }^{10}$.

Os encontros anteriores ao 72 estão disponíveis em arquivos no formato PDF e remontam desde o primeiro encontro. Foi feita uma coleta manual destes dados, restrita ao número total de participantes e à participação brasileira. A Figura 3 exibe um gráfico comparativo entre a participação global e a participação brasileira que somente começou no encontro 36, o segundo encontro realizado no ano de 1996.

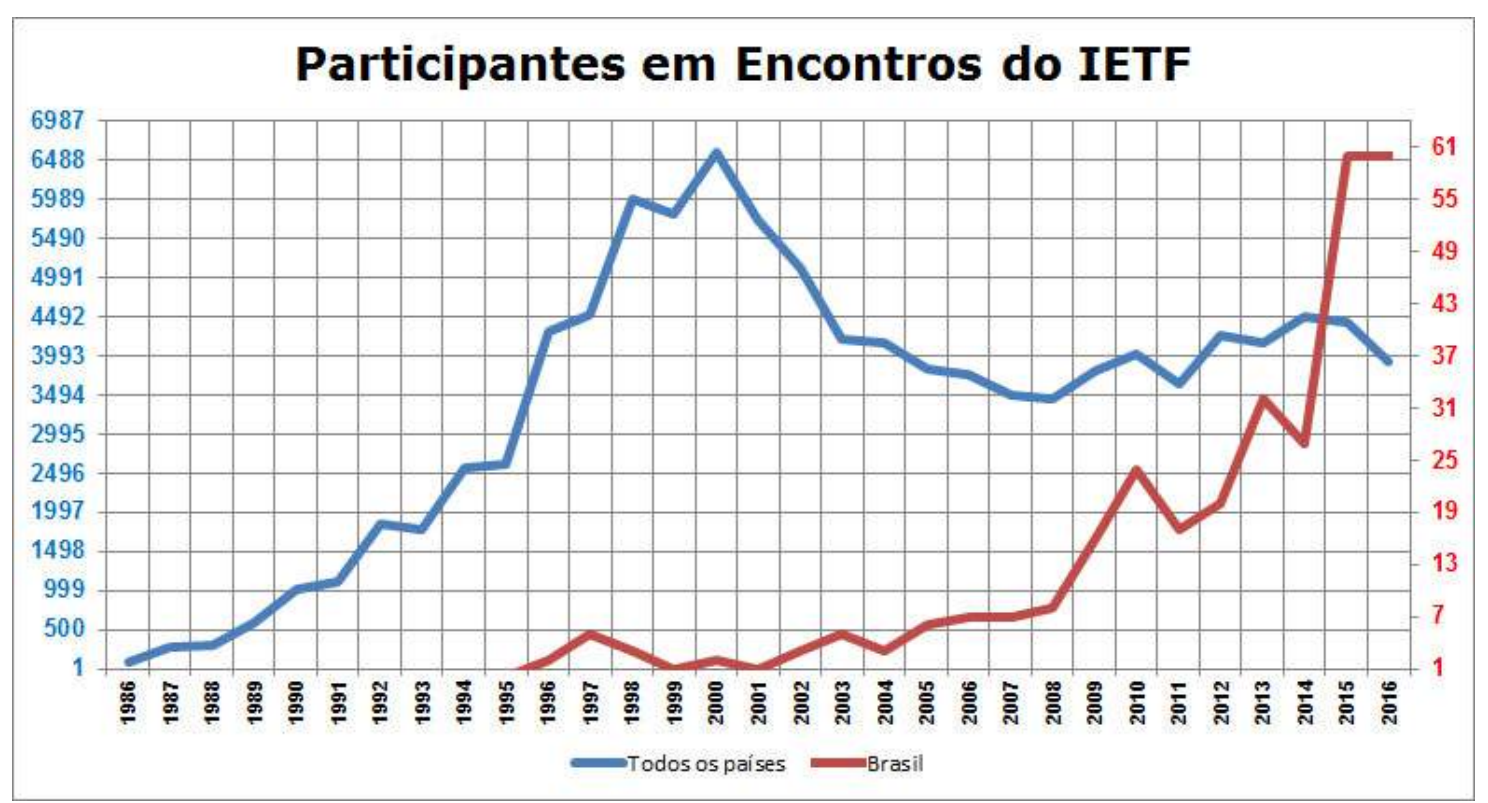

Figura 3. Participação brasileira desde o encontro 36 do IETF e a participação global, desde o primeiro encontro

\footnotetext{
${ }^{9} \mathrm{http} / / / \mathrm{www}$.internetsociety.org/who-makes-internet-work-internet-ecosystem

${ }^{10} \mathrm{http}: / /$ ietf.protocolos.net.br/meetings/ietf.php
} 
Esta figura indica um crescimento efetivo da participação brasileira, com maior intensidade a partir de 2009. Por outro lado, há um contraste bastante significativo entre a participação do Brasil e os outros países da América Latina como mostra a Figura 4.

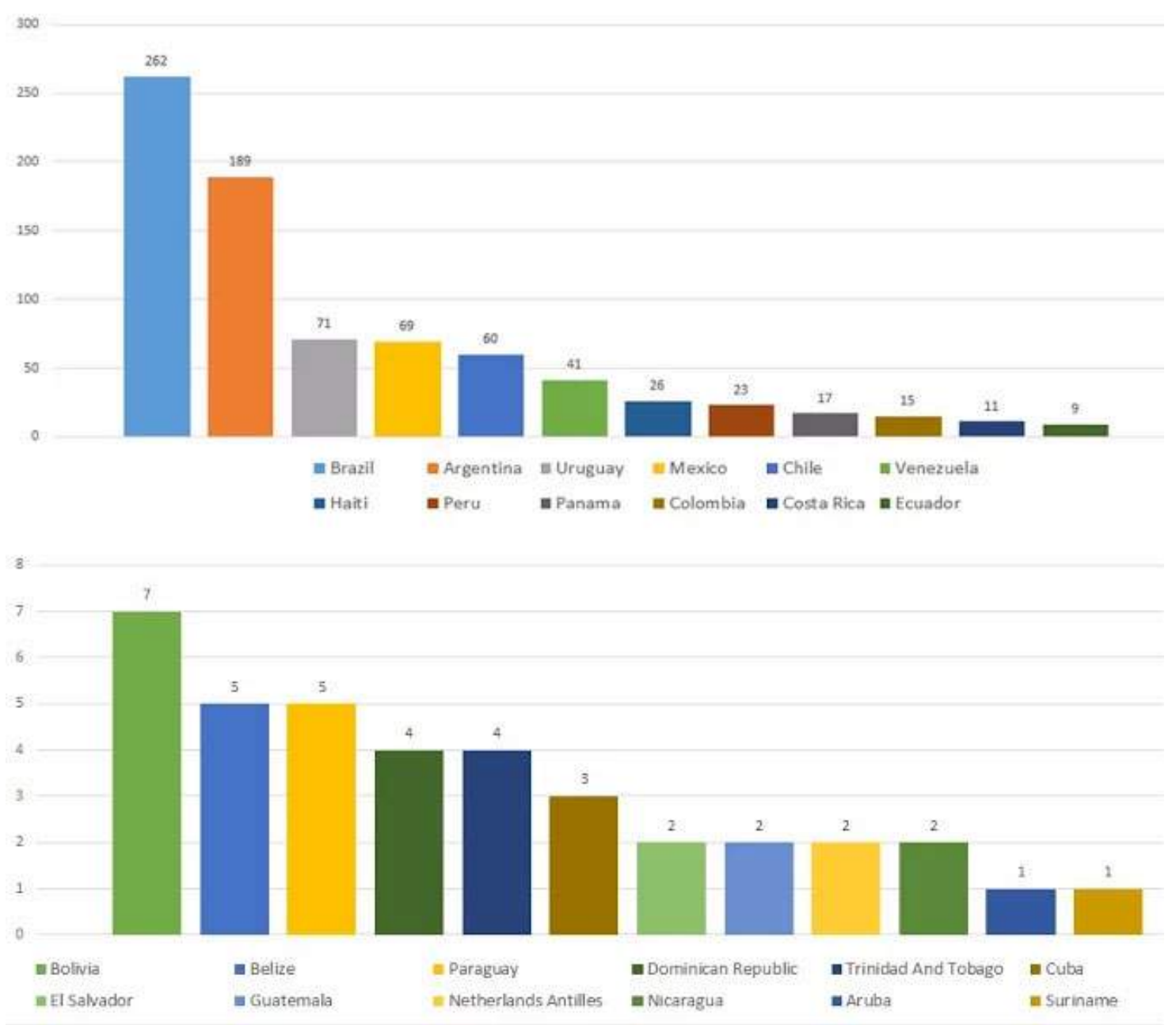

Figura 4. Número de participantes nos encontros do IETF, dos países da América Latina (encontros 72 a 97)

A quantidade de participantes não é exatamente uma surpresa se levar em conta a população do Brasil comparada com os outros países. O que chama a atenção é o crescimento com tendência exponencial, como fica evidente na Figura 5.

Tais argumentos e comparações, com base nos levantamentos preliminares indicaram a perspectiva de uma análise detalhada, o que recomendou o uso da metodologia aqui descrita.

Os dados, sob o ponto de vista dos resultados foram tratados, portanto, a partir da preocupação de entender as razões que contribuíram para este crescimento do Brasil, independente do que ocorreu nos outros países, alguns dos quais tiveram crescimento bem parecidos, como Argentina, Chile e México. As Figuras 6 e 7 exibem todos os países da América Latina e Caribe que participaram nas reuniões do IETF, no período considerado.

Chile e Argentina possuem mais afinidades no crescimento em relação ao Brasil, 


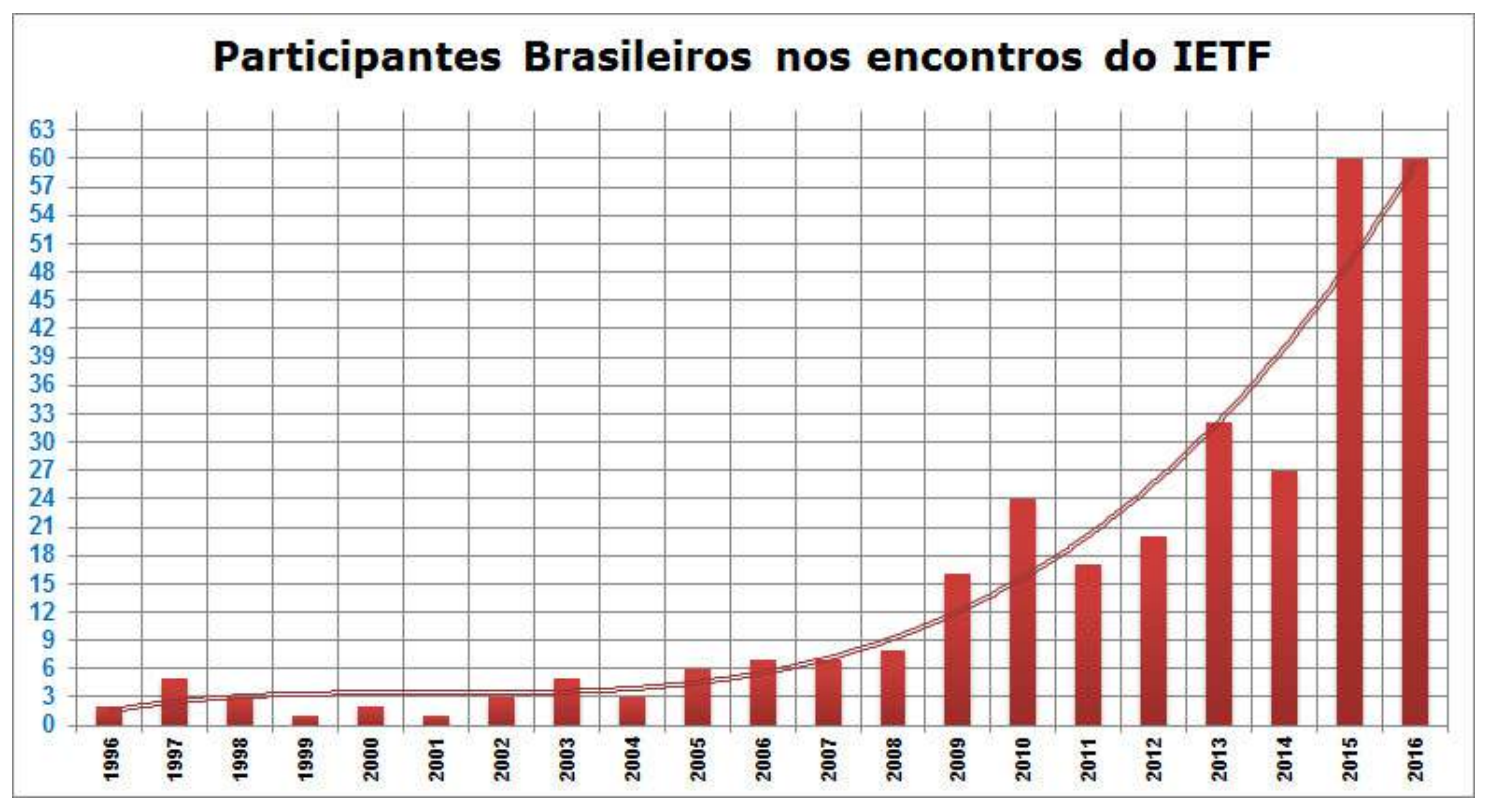

Figura 5. Evolução da participação do Brasil desde 1996 até 2016

seguido pelo México.

Os dados dos países são identificados pelos respectivos country code Top-Level Domain $^{11}$ (ccTLD), isto é, o domínio de topo ${ }^{12}$, que na Internet é usado ou reservado para um país ou um território dependente ${ }^{13}$. Alguns países na América Latina e Caribe, sobretudo no Caribe apresentam algumas distorções. É o caso de Netherlands Antilles - que aparece na Figura 4 -, cujo ccTLD é .an. Este território desapareceu em 2010. Ele representava algumas ilhas do Caribe ${ }^{14}$. Tais ilhas passaram a ter seus próprios ccTLDs mas com poucas ou quase nenhuma participação, devido a localização, geografia e características da população.

Os recursos da Internet para os países da América Latina e Caribe são gerenciados pelo LACNIC, um RIR acreditado pelo IANA. Em alguns casos, como o Brasil ${ }^{15}$ e México ${ }^{16}$, os recursos são geridos pelos National Internet Registry (NIR), mas sob orientação e cooperação do LACNIC.

\footnotetext{
${ }^{11}$ https://pt.wikipedia.org/wiki/CcTLD

${ }^{12} \mathrm{https} / / /$ pt.wikipedia.org/wiki/Dom\%C3\%ADnio_de_topo

${ }^{13} \mathrm{https}: / /$ pt.wikipedia.org/wiki/Lista_de_territ\%C3\%B3rios_dependentes

${ }^{14}$ https://en.wikipedia.org/wiki/Dissolution_of_the_Netherlands_Antilles

${ }^{15} \mathrm{http}: / /$ www.nic.br/

${ }^{16} \mathrm{http}: / /$ www.nicmexico.mx/
} 


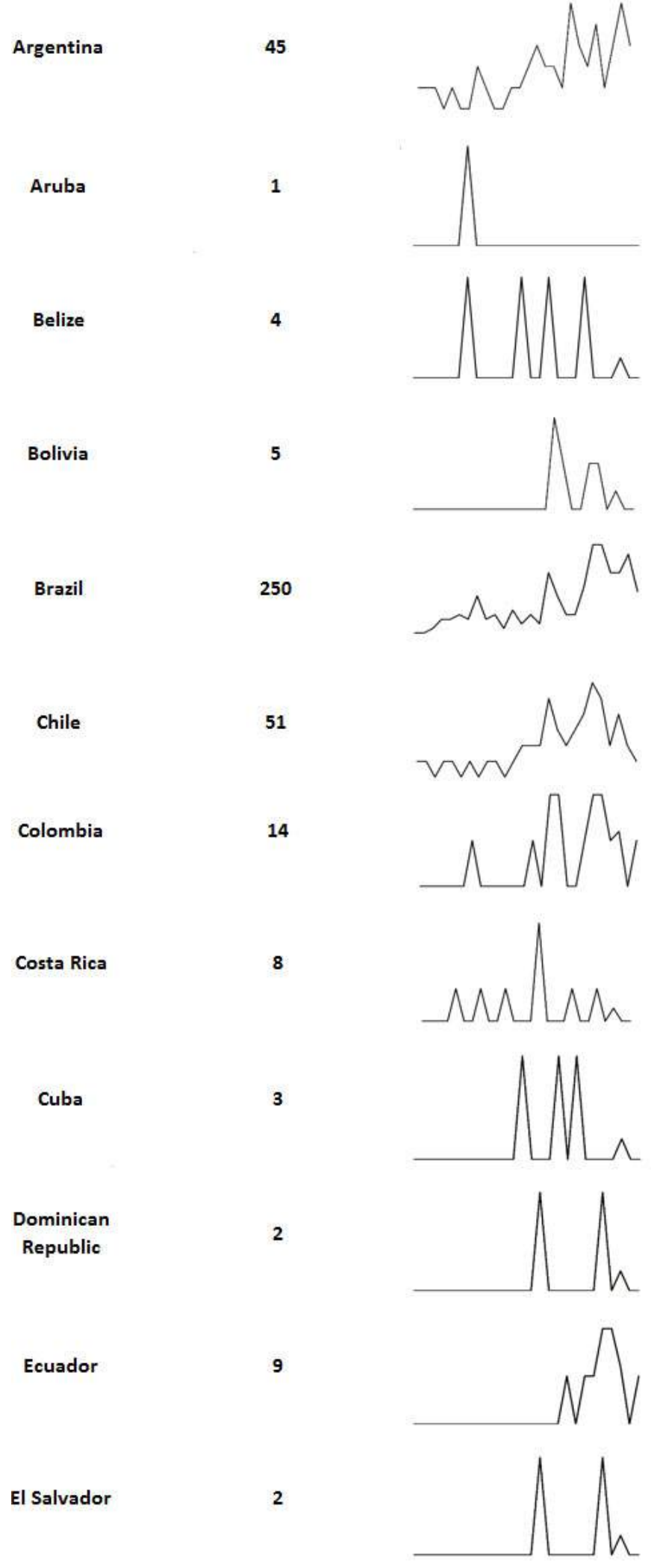

Figura 6. Países sob a supervisão do LACNIC, participação global e a curva de participação desde o IETF 72 - Parte I 


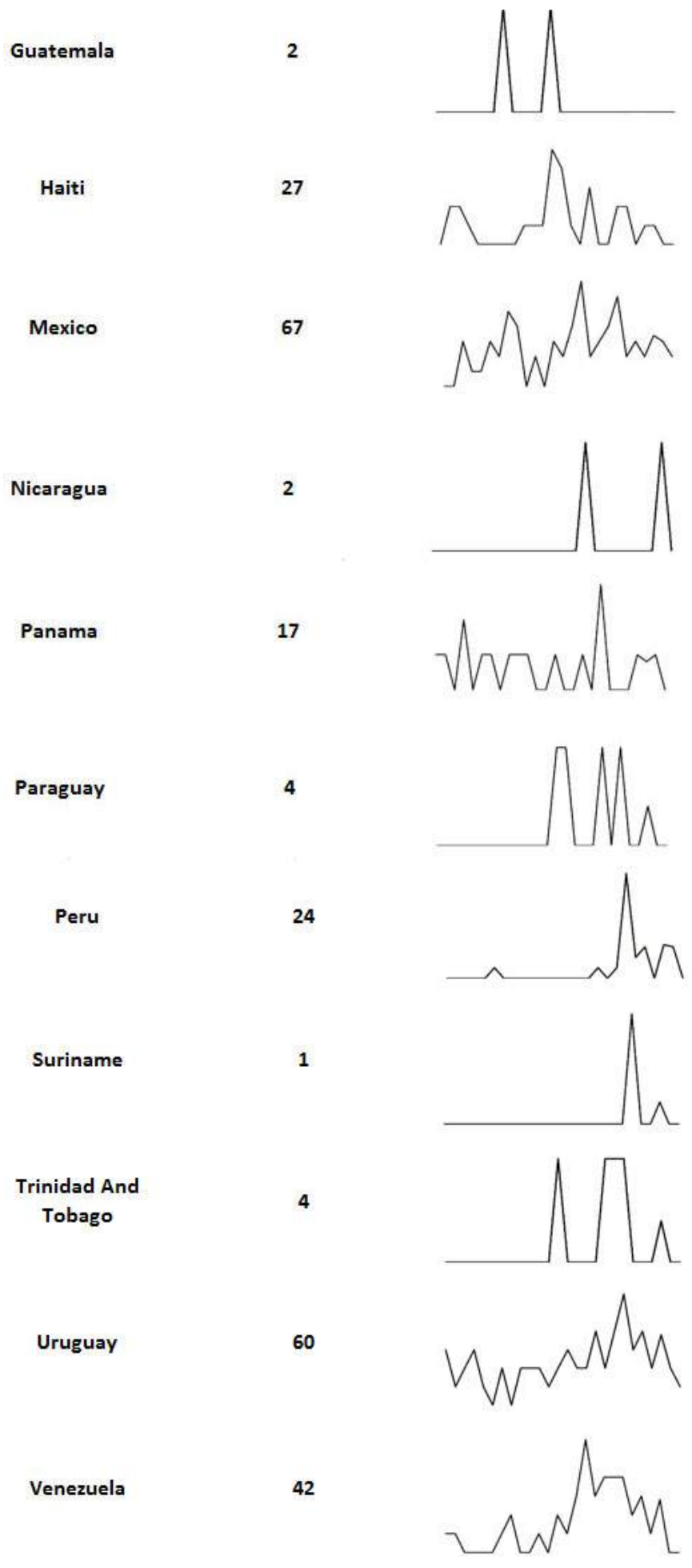

Figura 7. Países sob a supervisão do LACNIC, participação global e a curva de participação desde o IETF 72 - Parte II

A seguir, apresentamos e descrevemos os dados tratados considerando, especificamente, a América Latina e Caribe, com ênfase no Brasil. 


\section{A região do LACNIC}

O LACNIC é responsável pela designação e administração dos recursos de numeração da Internet (IPv4, IPv6), Números Autônomos e Resolução Inversa, entre outros recursos para a região da América Latina e o Caribe. É um dos cinco Registros Regionais da Internet no mundo ${ }^{17}$.

Complementarmente às Figuras 4 e ?? , a Figura 8 exibe um trecho do mapa mundial com os territórios sob o controle do LACNIC em relação a seus recursos da Internet. Detalhes sobre os países da América Latina e Caribe nos encontros do IETF podem ser vistos na versão interativa do mapa ${ }^{18}$.

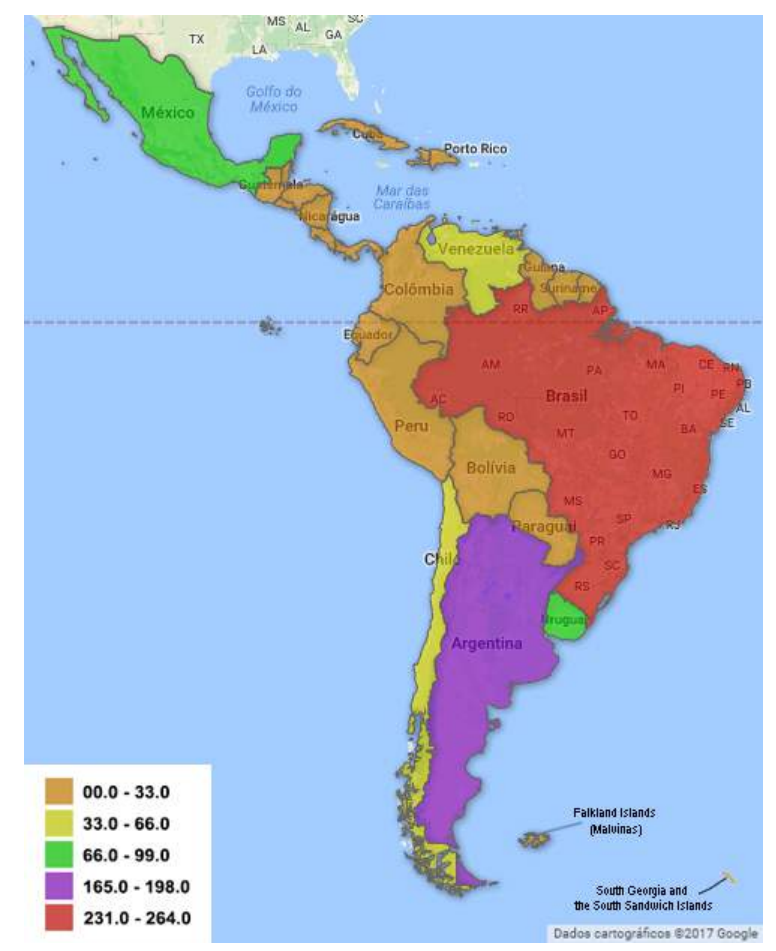

Figura 8. Mapa interativo da participação dos países sob o controle dos recursos de Internet, do LACNIC. Fonte e mapa interativo: https://goo.gl/V4cOvi

Cinco países nunca levaram participantes para reuniões do IETF: South Georgia and The South Sandwich Islands, Honduras, Guiana, Guiana Francesa e Falkland Islands (Malvinas). Honduras, coincidentemente está com duas participações no IETF 98. O IETF 98 não foi considerado neste trabalho, e as participações da região do LACNIC estão visíveis na Tabela 1.

\section{Participação do Brasil}

A Figura 5 exibe a participação do Brasil nos encontros do IETF (cada coluna representa a soma dos três encontros anuais), de 1996 até 2016. Como pode-se observar, a partir se 2009, o Brasil começou a aumentar a quantidade de participantes.

\footnotetext{
${ }^{17}$ http://www.lacnic.net/pt/web/lacnic/acerca-lacnic

${ }^{18}$ https://goo.gl/V4cOvi
} 
Tabela 1. Participação da região do LACNIC no IETF $98 . \quad$ Fonte: http://ietf.protocolos.net.br/meetings/latin98.php

\begin{tabular}{|l|c|r|r|r|}
\hline \multicolumn{1}{|c|}{ País } & ccTLD & \multicolumn{1}{c|}{$\#$} & \% Total & \% RIR \\
\hline Argentina & AR & 4 & 0,31 & 10,81 \\
\hline Brasil & BR & 12 & 0,31 & 10,81 \\
\hline Chile & CL & 5 & 0,38 & 13,51 \\
\hline Colombia & CO & 2 & 0,15 & 5,41 \\
\hline Guiana & GY & 1 & 0,08 & 2,70 \\
\hline Honduras & HN & 2 & 0,15 & 5,41 \\
\hline Haiti & HT & 1 & 0,08 & 2,70 \\
\hline Mexico & MX & 4 & 0,31 & 10,81 \\
\hline Panama & PA & 1 & 0,08 & 2,70 \\
\hline Peru & 1 & 0,08 & 2,70 & \\
\hline Uruguai & UY & 3 & 0,23 & 8,11 \\
\hline Venezuela & VE & 1 & 0,08 & 2,70 \\
\hline \multicolumn{2}{|c|}{ Total } & $\mathbf{3 7}$ & $\mathbf{2 , 8 5}$ & $\mathbf{1 0 0 , 0 0}$ \\
\hline \multicolumn{5}{|c|}{} \\
\hline
\end{tabular}

Na subseção 5.1, serão discutidas algumas ações realizadas no Brasil que contribuíram para este resultado.

\subsection{Ações no Brasil}

Algumas ações tem sido desenvolvidas no Brasil com o objetivo de estimular a participação (mais efetiva) brasileira no processo de padronização de protocolos da Internet.

Ação 1: A partir de 2010 foram elaborados diversos documentos e palestas em instituições e em eventos brasileiros com o objetivo de despertar o interesse pelo IETF. Ativas nestas iniciativas estavam a Universidade Federal de Alagoas, Universidade de Pernambuco e Universidade Presbiteriana Mackenzie.

Ação 2: No início de 2013 (maio), foi criada a lista IETF-LAC ${ }^{19}$. O objetivo da lista é agregar e estimular iniciativas na América Latina e Caribe, relacionadas com o IETF e IRTF.

Ação 3: Em 2013, O Tao do IETF: Guia destinado aos novos participantes do Internet Engineering Task Force ${ }^{20}$ foi traduzido para as línguas espanhola e portuguesa.

Ação 4: Em 2014 aconteceu o I Workshop pré-IETF ${ }^{21}$. Nos anos seguintes, associados ao Congresso da Sociedade Brasileira de Computação (SBC) foram realizadas as segunda (2015), terceira (2016) e a quarta (2017) edições. O principal objetivo deste evento é expandir a presença da América Latina e Caribe nos encontros do IETF, além de proporcionar um ambiente para que as habilidades de técnicos, estudantes e pesquisadores pudessem ser demonstradas, antecipando-se aos três encontros anuais do IETF. Como estímulo à participação, a partir da quarta edição (2017) três prêmios serão concedidos aos três mais relevantes trabalhos. O primeiro prêmio é uma participação no próximo encontro do IETF, o segundo prêmio

\footnotetext{
${ }^{19}$ https://mail.lacnic.net/mailman/listinfo/ietf-lac

${ }^{20} \mathrm{https}$ ://www.ietf.org/tao-translated-br.html

${ }^{21} \mathrm{http} / / /$ www.cudi.edu.mx/eventos/i-workshop-pre-ietf
} 
é uma participação no próximo encontro do LACNIC e o terceiro prêmio é uma participação na Semana de Infraestrutura da Internet promovida pelo CGI.br.

Ação 5: Em 2014 foi criado um grupo no Facebook ${ }^{22}$, como apoio complementar à lista IETF-LAC.

Ação 6: Em 2014, sob o patrocínio do Comitê Gestor da Internet Brasileira (CGI.br), foram produzidos 30.000 exemplares de um livro contendo O Tao do IETF em espanhol, inglês e portugues [Braga et al. 2014]. Esta ação proporcionou a distribuição do livro e consequentemente contribuiu para a difusão do IETF na América Latina, Caribe e em alguns países e eventos da África (Cabo Verde, Guiné Equatorial, Moçambique e São Tomé e Príncipe). Adicionalmente está disponível uma versão em $\mathrm{PDF}^{23}$.

Ação 7: Em 2014, o CGI.br lançou uma chamada pública para participação em grupos de trabalhos e reuniões do IETF/IRTF, com o objetivo de ampliar ainda mais a participação brasileira ${ }^{24}$. O edital contemplou três projetos, totalizando oito pesquisadores brasileiros, além de um contemplado na modalidade de profissionais vinculados à indústria de redes nacional ${ }^{25}$. Na seção 6 há uma descrição detalhada deste projeto.

Ação 8: Em 2016 surgiu uma iniciativa embrionária denominada Internet Academy, que tem como objetivo revisitar o processo de ensino de redes, especialmente das tecnologias de Internet, e propor melhores estratégias e metodologias neste contexto. A Internet Academy está fortemente relacionada com o IETF, uma vez que também está preocupada com a formação de profissionais voluntários que sejam capazes de auxiliar de forma sistemática nas atividades do IETF.

Paralelamente à essas ações brasileiras, ressalta-se uma ação internacional promovida pela ISOC, denominada ISOC Internet Leadership, que financia a participação de estudantes, docentes e profissionais de países em desenvolvimento nos encontros do IETF. O programa existe desde 2007 e fornece inscrição para o evento, passagem, hospedagem e ajuda de custo para os bolsistas selecionados. Nos últimos seis encontros, o Brasil esteve representado através de quinze bolsas, até o IETF97, como pode ser visto na Tabela 2.

Tabela 2. Bolsistas brasileiros no IETF. Fonte: Internet Society

\begin{tabular}{|c|c|}
\hline Encontro & Bolsistas \\
\hline \hline 93 & 3 \\
\hline 94 & 4 \\
\hline 95 & 4 \\
\hline 96 & 2 \\
\hline 97 & 2 \\
\hline
\end{tabular}

\section{Participação brasileira no desenvolvimento de documentos}

Apesar da importância do Brasil no contexto de redes de computadores, a participação de brasileiros no desenvolvimento de documentos para o IETF ainda é significativamente

\footnotetext{
${ }^{22}$ https://www.facebook.com/groups/1612210999058155/

${ }^{23} \mathrm{http} / / /$ cgi.br/media/docs/publicacoes/1/o-livro-do-ietf.pdf

${ }^{24}$ http://cgi.br/editais/ver/2.pdf

${ }^{25} \mathrm{http}$ ://www.cgi.br/noticia/notas/cgi-br-divulga-resultado-da-selecao-publica-para-o-ietf-e-irtf/
} 
pequena. Considerando o melhor conhecimento dos autores, podem ser citadas as seguintes participações em documentos para o IETF/IRTF: Antônio Moreiras (Núcleo de Informação e Coordenação do Ponto BR - NIC.BR), Edwin Cordeiro (Technical University of Munich), Frederico Neves (NIC.BR), Julião Braga (Universidade Presbiteriana Mackenzie e University of Saskatchewan).

Dificuldades financeiras podem ser citadas para esta escassez de participações, já que as oportunidades de financiamento são menores do que aquelas oferecidas em outros países. Neste contexto, a Chamada Pública 0001/2014, financiada pelo CGI.br, proporciona um auxílio significativo para o desenvolvimento de atividades conjuntas entre o IETF/IRTF e membros de instituições de pesquisa e fabricantes de equipamentos baseados no Brasil.

Esta iniciativa selecionou 4 participações nas reuniões do IETF: o projeto "Utilização de Tecnologia Par-a-Par para Controle Autonômico Distribuídos de Sondas de Monitoramento em Redes de Computadores", dos autores Jéferson Nobre e Lisandro Granville, representando a Universidade Federal do Rio Grande do Sul (UFRGS); o projeto "Metrologia e ciência de redes aplicadas à Internet", dos autores Klaus Wehmuth e Artur Ziviani, representando o Laboratório Nacional de Computação Científica (LNCC/MCTI); e o projeto "Redes Definidas por Software considerando cenários com Virtualização de Funções da Rede", dos autores Marcelo Santos, Felipe Lopes, Leonidas Lima e Stênio Fernandes, representando a Universidade Federal de Pernambuco (UFPE). Além destes, também foi selecionado o apoio à participação de Denis Michellis, na modalidade de profissionais vinculados à indústria de redes nacional, funcionário da instituição Telefônica Brasil SA.

A participação dos pesquisadores dos projetos contemplados no IETF através desta chamada foi benéfica para os próprios pesquisadores assim como para a inserção brasileira no IETF. A experiência ganha em tal participação tem sido compartilhada nas instituições dos pesquisadores, o que pode auxiliar no desenvolvimento e padronização de protocolos para a Internet.

\section{Considerações finais}

Há diversas outras fontes de recursos para participações, além daquelas descritas acima. $\mathrm{O}$ IV Workshop pré IETF, com apoio do capítulo brasileiro da ISOC Brasil está implementando pela primeira vez uma premiação que envolve a participação de autores de trabalhos premiados, no próximo encontro do IETF, após a realização do Workshop. Além disso, o Applied Networking Research Workshop (ANWR) ${ }^{26}$, uma iniciativa do IRTF, é outro exemplo de fonte de recursos que fomentam a dupla participação: no próprio ANWR e no IETF que se realiza na sequência. No primeiro encontro do ANWR houve, pelo menos, três participações brasileiras, sendo duas delas estudantes com bolsa do ANWR.

Tais oportunidades devem ser divulgadas sem restrições com o objetivo de incentivar estudantes, professores e profissionais que trabalham na área. Adicionalmente, iniciativas como as da ISOC Brasil, CGI.br devem ser estimuladas e ampliadas para a região do LACNIC. A experiência provocada por iniciativa do CGI.br, descrita na seção (6) deve ser estimulada a se repetir, pois ela permitiu a ocupação de espaços importan-

\footnotetext{
${ }^{26}$ https://irtf.org/anrw/
} 
tes, de liderança, nas atividades que gravitam em torno do IETF, IRTF, ISOC e outras instituições associadas ${ }^{27}$.

Parece ter chegado o momento de provocar a efetiva participação de voluntários brasileiros no desenvolvimento de documentos para o IETF e/ou IRTF. Neste sentido, a Internet Academy ${ }^{28}$ (IA) reproduz propostas interessantes. Além de ações de atualização da aprendizagem de redes de longa distância projeta-se um treinamento focado no voluntariado efetivo, em particular, do IETF. Adicionalmente, a IA está propondo diversas edições de "hackathon"durante eventos importantes no Brasil e no restante da região do LACNIC.

\section{Referências}

Alvestrand, H. (2004). A Mission Statement for the IETF. BCP 95, RFC Editor.

Bradner, S. O. (1996). The Internet Standards Process - Revision 3. BCP 9, RFC Editor. http: //www.rfc-editor.org/rfc/rfc2026.txt.

Braga, J., Granville, L. Z., O'Flaherty, C., and Moreiras, A. M. (2014). O Livro do IETF. CGI.br, São Paulo, 1 edition.

Braga, J. and Omar, N. (2014). Semantic Repository in Internet Infrastructure Knowledge Domain: Methodology. In Anais do CSBC 2014, pages 2179-2184, Brasília, DF.

Hoffman, P. and Harris, S. (2006). The Tao of IETF - A Novice's Guide to the Internet Engineering Task Force. RFC 4677, RFC Editor.

Huizer, E. (1996). Ietf-isoc relationship. RFC 2031, RFC Editor.

Leiner, B. M., Cerf, V. G., Clark, D. D., Kahn, R. E., Kleinrock, L., Lynch, D. C., Postel, J., Roberts, L. G., and Wolff, S. (2009). A brief history of the Internet. ACM SIGCOMM Computer Communication Review, 39(5):22-31.

Postel, J. (1993). Instructions to RFC Authors. RFC 1543, RFC Editor.

Simonelis, A. (2005). A concise guide to the major internet bodies. Ubiquity, 2005(February):2-2.

Weinrib, A. and Postel, J. (1996). IRTF Research Group Guidelines and Procedures. BCP 8, RFC Editor. http: / / www.rfc-editor.org/rfc/rfc2014.txt.

\footnotetext{
${ }^{27}$ https://sites.google.com/site/internetresearchlabs/

${ }^{28} \mathrm{http}: / /$ www.internetacademy.org.br
} 